\title{
Effects of octreotide and allopurinol on liver histology and functions in surgical jaundice: An experimental study
}

Effects of octreotide and allopurinol in surgical jaundice.

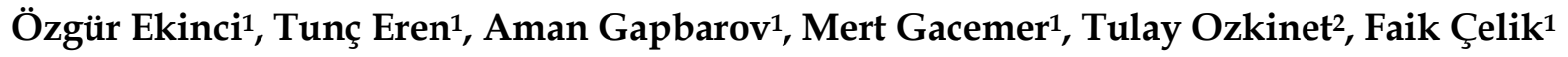

\begin{abstract}
Aim: Mechanical jaundice may lead to adverse metabolic effects in surgical patients. Our aim was to investigate the effects of octreotid and allopurinol on liver histology and functions in rats with experimantally created mechanical jaundice.

Methods: The subjects were divided into five main groups. The rats in Group I (Control) received no interventions. In Group II, common bile duct ligation (CBDL) was performed. In Group III, the same ligation procedure was performed followed by the administration of octreotid. In Group IV, the rats received allopurinol following CBDL and in Group V (Sham), the common bile duct was dissected and left intact. After sacrification of each rat on the seventh postoperative day, blood samples were collected for serum hematocrit, direct bilirubin, alanine aminotransferase (ALT), aspartate aminotransferase (AST), amylase, and albumin measurements in addition to the histopathologic examinations of the collected tissue specimens from the liver, terminal ileum, and pancreas. According to statistical analysis, a value of $\mathrm{p}<0.05$ was considered as significant.

Results: The direct bilirubin, AST and ALT levels were detected to be significantly elevated in the groups II, III and IV, in which CBDL was performed $(\mathrm{p}<0.05)$. While significant reductions of serum direct bilirubin, AST and ALT levels were detected in the octreotide group, these parameters were significantly elevated in the allopurinol group ( $p<0.05$ and $p<0.05$, respectively). There was no significant difference of hematocrit and albumine levels among the study groups $(\mathrm{p}>0.05)$. Additionally, in the allopurinol group four rats were found to have increased serum amylase levels and the histopathologic evaluations of their pancreatic samples revealed findings of pancreatitis. According to the histopathologic examinations, significant alterations were detected between the control and sham groups (groups I, V) and the CBDL groups (groups II, III, IV). However, these histopathological findings revealed no significant differences within the subgroup of subjects including CBDL alone (Group II), and CBDL along with the administration of either octreotide (Group III), or allopurinol (Group IV).

Conclusion: The use of octreotide in mehanical jaundice may have protective metabolic effects. Further studies are considered necessary to be carried to support these findings.
\end{abstract}

Keywords: jaundice, octreotide, allopurinol, general surgery.

*Corresponding Author: Tunç Eren

$\equiv \triangle$ E-mail: $\underline{\text { drtunceren@ gmail.com }}$

${ }^{I}$ Department of General Surgery, Istanbul Medeniyet University, Faculty of Medicine, Goztepe Training \& Research Hospital, Istanbul, Turkey

${ }^{2}$ Department of Pathology, Istanbul Medeniyet University, Faculty of Medicine, Goztepe Training \& Research Hospital, Istanbul, Turkey. 


\section{Full Text}

\section{Introduction}

The liver secretes a daily average of 1-1.5 liters of bile which is passed into the intestines through the bile ducts. Obstruction of bile flow to the duodenum at any reasons (stones, tumors, stenosis, inflammation, external compression) results in the accumulation of bile in the extra- and intrahepatic bile ducts as this congestion is defined as mechanical (surgical) jaundice. The bilirubin mechanism fails in cases of mechanical jaundice leading to elevated direct bilirubin levels which is associated with specific symptoms \& signs including yellowing of the scleras, darkening of urine, pale stool and pruritus [1]. The most important secondary effect of mechanical jaundice is the development of infection proximally to the obstruction which may lead to ascending cholangitis and the most common causative microorganism is Escherichia coli $[2,3]$.

The discovery of somatostatin, which is a natural pancreatic hormone, and the studies on its effects and role in several diseases lead to the development of synthetic somatostatin analogs such as octreotid (Sandostatin ${ }^{\circledR}$, SMS 201-995) [4]. Octreotid has a wide indication spectrum especially in enterocutaneous fistulas, portal hypertension and acute pancreatitis.

Allopurinol, which is a xanthine oxidase inhibitor, is conventionaly used to treat gout and it is also used in other conditions for supportive treatment such as to reduce the adverse effects of chemotherapy given that the enzyme xanthine oxidase is effective on the purine metabolism $[5,6]$. The enzyme xanthine oxidase enzyme is predominantly found in the liver $[7,8]$.

Mechanical jaundice may lead to adverse metabolic effects and general anesthesia primarily poses an increased risk in these cases. Pre-existing comorbidities including diabetes mellitus, malignancy and infections, significantly increase morbidity \& mortality rates. In this study, we aimed to investigate the effects of octreotid and allopurinol on liver histology and functions in rats with experimantally created mechanical jaundice.

\section{Methods}

This study was carried out at Istanbul University, Experimental Medicine Research Institute, Laboratory Animal Science Department with the approval of the Animal Experiments Local Ethics Committee of Istanbul University. Fifty male Wistar Albino rats, with weights between 150 and 200 grams, were used in the study. All subjects were fed with standard laboratory food and water.

The subjects were divided into five main groups each of which consisted of 10 rats. The groups were set as; Group I: Control 
(C), Group II: Common bile duct ligation (CBDL), Group III: CBDL + octreotid $(\mathrm{CBDL}+\mathrm{O})$, Group IV: CBDL + Allopurinol (CBDL+A), and Group V: Sham laparotomy (Sh). The rats in Group
$C$ received no interventions. In Group II (CBDL), a laparotomy was performed under ether anesthesia after shaving of the abdomen and the common bile duct was ligated with 3-0 silk (Figure 1).

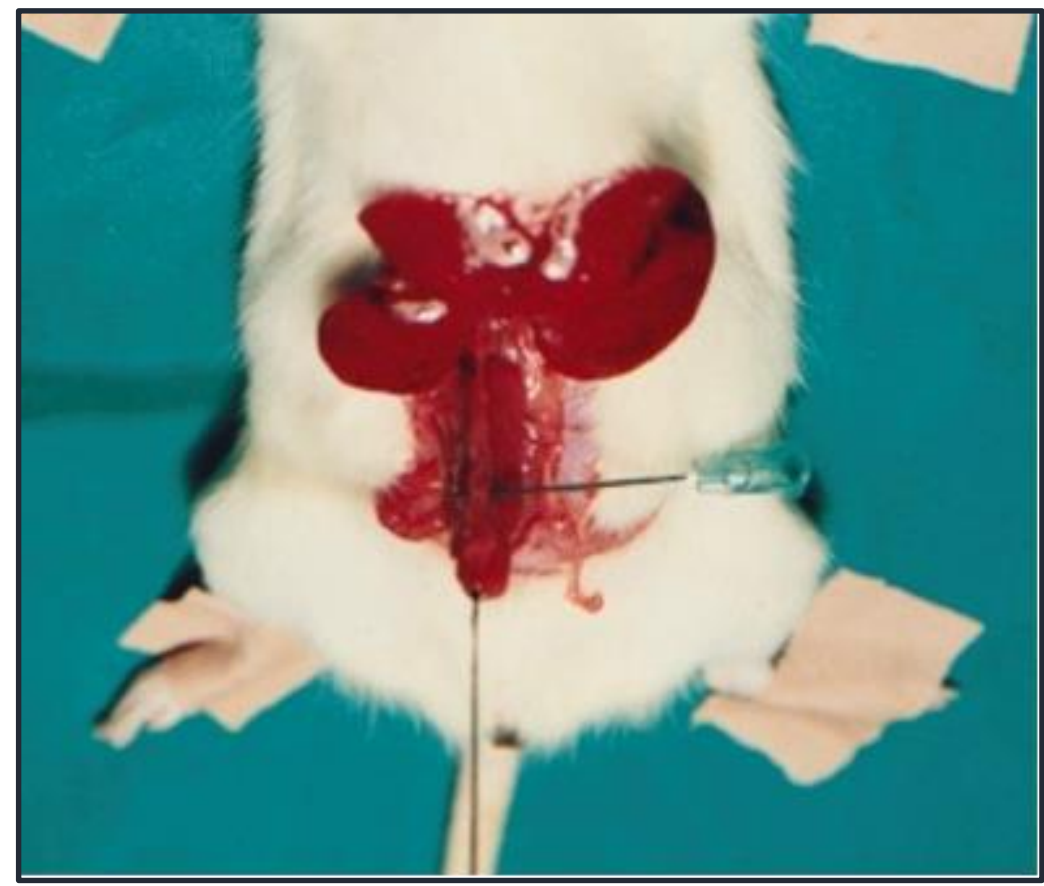

Figure 1. The surgical preparation of the common bile duct.

In Group III $(\mathrm{CBDL}+\mathrm{O})$, the same ligation procedure was performed followed by the subcutaneous administration of octreotid at a dose of $0.1 \mathrm{mg} / \mathrm{kg}$ for seven days postoperatively before the rats were sacrificed. In Group IV (CBDL+A), the rats received $0.1 \mathrm{mg} / \mathrm{kg}$ of allopurinol enterally through a polyethylene nasogastric tube for seven days postoperatively before sacrification following the bile duct ligation procedure. In Group V (Sh), the common bile duct was dissected, seperated from the surrounding tissue and left intact without ligation. All rats had their laparotomy incision closure via two layered continuous suturing with use of 3-0 prolene.

The development of mechanical jaundice was verified clinically by acholic stool, urine darkening and biochemical findings in the rats that underwent common bile duct ligation. Following the sacrification of each rat on the seventh postoperative day, a laparotomy was created for the subjects in Group I (C), and a relaparotomy was performed in all subjects of the remaining study groups. The rats blood samples were collected 
via cardiac puncture for the measurements of serum hematocrit (Hct), direct bilirubin, alanine aminotransferase (ALT), aspartate aminotransferase (AST), amylase and albumine levels. After abdominal exploration, the tissue specimens collected from the liver, terminal ileum, and pancreas were fixated in $10 \%$ formaline solution and the histopathologic examinations were carried out by a single pathologist in our institution.

Additional to the descriptive statistical methods (mean, standard deviation, median, frequency, ratio, minimum, maximum), the analysis of variance (ANOVA) was used to compare quantitative data between the groups and for the comparison of qualitative data, the Kruskal-Wallis test was used. A value of $\mathrm{p}<0.05$ was considered as statistically significant.

\section{Results}

The direct bilirubin, AST and ALT levels were detected to be significantly elevated in Groups II (CBDL), III (CBDL+O) and IV (CBDL+A) when compared with Groups I $(C)$ and V $(S h)(p<0.05)$. While statistically significant reductions of serum direct bilirubin, AST, and ALT levels were detected in Group III $(\mathrm{CBDL}+\mathrm{O})$ when compared with Group II (CBDL), these parameters were significantly elevated in Group IV $(\mathrm{CBDL}+\mathrm{A})$ when compared to the remaining study groups $(\mathrm{p}<0.05$ and $\mathrm{p}<0.05$, respectively) (Table 1 ).

There was no statistically significant difference of Hct and albumine levels among the study groups $(\mathrm{p}>0.05)$ (Table 1).

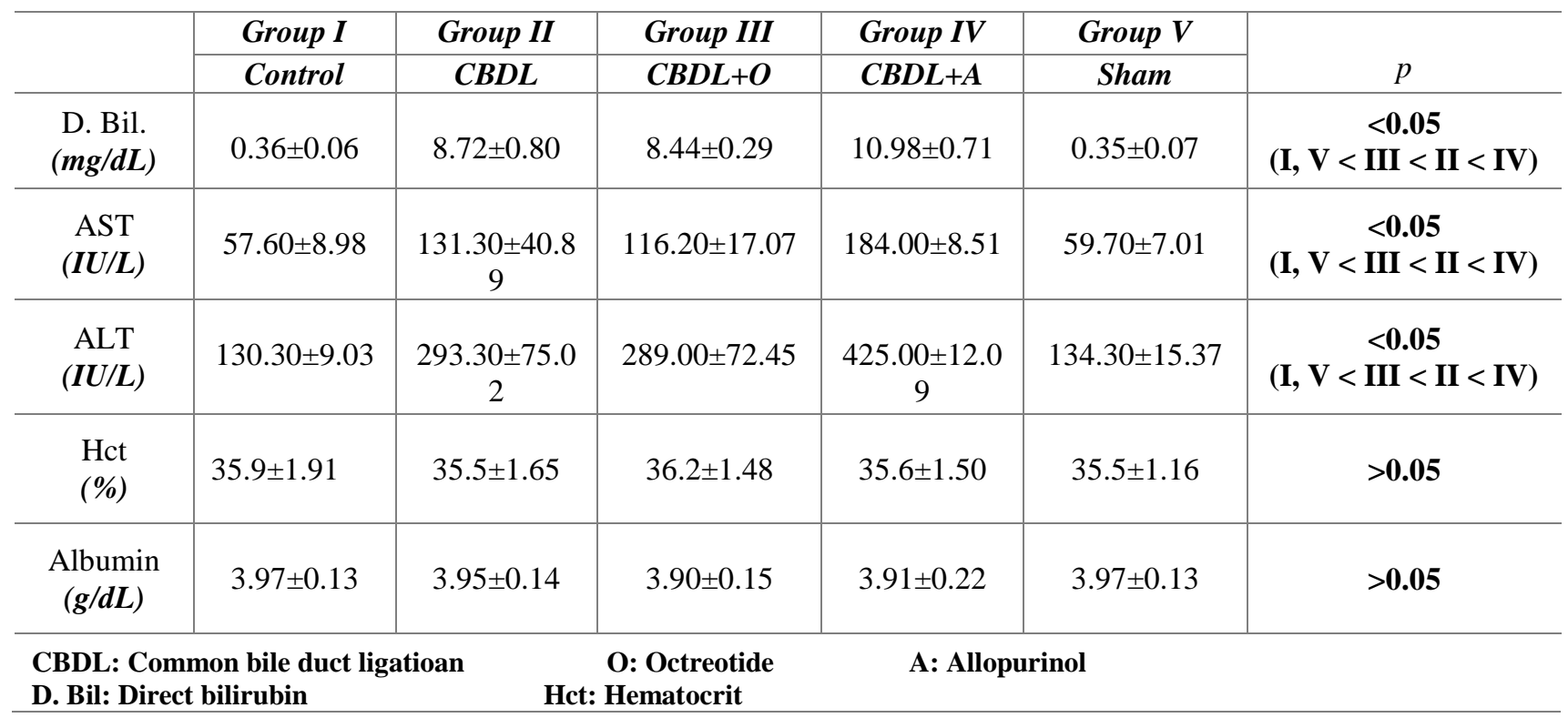


Table 1. The comparisons of direct bilirubin, AST and ALT levels among the study groups.

No significant difference was detected according to serum amylase levels among the study groups except Group IV $(\mathrm{CBDL}+\mathrm{A})$ as four rats were found to have increased serum amylase levels in this group and the histopathologic evaluations of the pancreatic samples of these four subjects revealed findings of pancreatitis (Table 2).

\section{Group IV $(\boldsymbol{C B D L}+\boldsymbol{A})$}

\begin{tabular}{|c|c|}
\hline $\begin{array}{c}\text { Subject no. } \\
(\#)\end{array}$ & $\begin{array}{c}\text { Amylase } \\
(I U / m l)\end{array}$ \\
\hline $\mathbf{1}$ & 60 \\
\hline $\mathbf{2}$ & 65 \\
\hline $\mathbf{3}$ & 70 \\
\hline $\mathbf{4}$ & $\mathbf{4 5 5}$ \\
\hline $\mathbf{5}$ & 70 \\
\hline $\mathbf{6}$ & $\mathbf{4 5 8}$ \\
\hline $\mathbf{7}$ & $\mathbf{6 0 0}$ \\
\hline $\mathbf{8}$ & 60 \\
\hline $\mathbf{9}$ & 61 \\
\hline $\mathbf{1 0}$ & $\mathbf{4 7 0}$ \\
\hline
\end{tabular}

CBDL: Common bile duct ligation

A: Allopurinol

Table 2. Summary of the serum amylase measurements in Group IV $(C B D L+A)$ showing significantly elevated levels in subjects 4, 6, 7 and 10.

According to the examinations of the tissue samples collected from the liver, terminal ileum, and pancreas, significant histopathological alterations were detected in Groups II (CBDL), III $(\mathrm{CBDL}+\mathrm{O})$ and IV $(\mathrm{CBDL}+\mathrm{A})$ when compared with the Groups I (C) and V (Sh). However, these histopathological findings of the tissue samples revealed no significant differences within the subgroup of subjects having undergone bile duct ligation when compared among Groups II (CBDL), III (CBDL+O) and IV $(\mathrm{CBDL}+\mathrm{A})$. 
In the subgroup of subjects having undergone bile duct ligation, the histopathological alterations of the terminal ileal specimens included flattening of the mucosa, heavy cellular infiltration of lymphocytes, plasma cells, neutrophils and eosinophils in the lamina propria and serosa as well as mesothelial cellular proliferations. In the intestinal mucosa, the glands were visualized to be partially atrophic. Additionally, in this subroup, the histopathological alternations of the liver sections included dilation of the bile ducts and vessels, endothelial proliferation in the vessels lumens, periductal mesenchymal cell proliferation, lymphocyte, plasma cell and leukocyte infiltration, and endothelial proliferation. Vacuolization of hepatocytes and degenerative changes were remarkable. Dilation and erythrocyte accumulation were observed in the sinusoids as well (Figure 2).

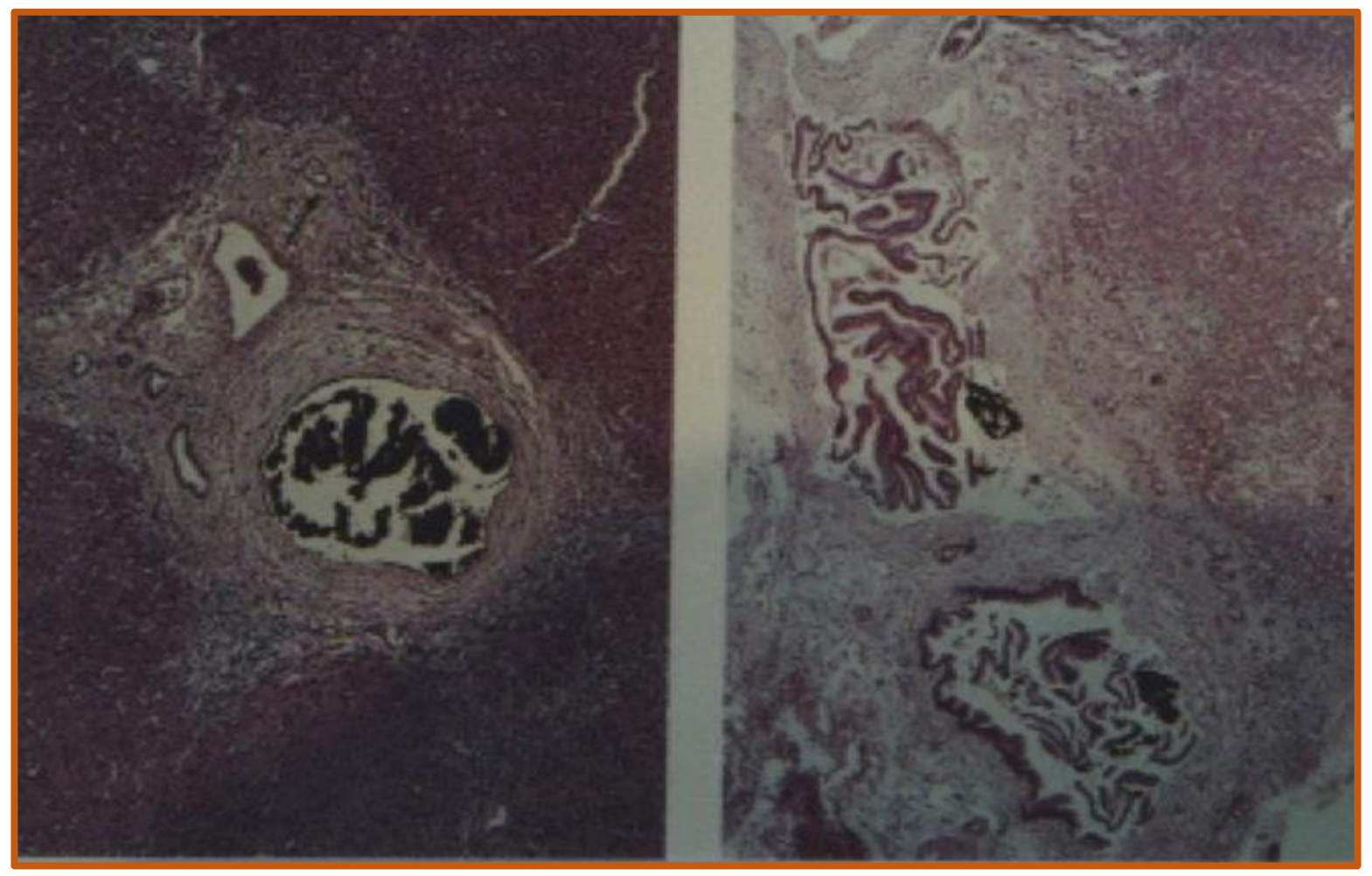

Figure 2. Histopathological section of a liver specimen revealing erythrocyte accumulation within the dilated sinusoids. 


\section{Discussion}

Postoperative hemorrhage, sepsis and organ failure are seen more commonly in surgical patients with mechanical jaundice $[9,10]$. According to the study of Pitt et al, risk factors for biliary surgery included age, presence of malignancy, anemia, sepsis, leukocytosis, and elevated serum bilirubin levels higher than $10 \mathrm{mg} / \mathrm{dL}$ [11]. Consequences such as anemia, sepsis or elevated bilirubin levels higher than $10 \mathrm{mg} / \mathrm{dL}$ were not detected in our study group.

Blamey et al reported the serum albumine level as an important factor to estimate biliary tract risk analysis [12]. In the present study, no difference was found among the study groups regarding albumine when compared with the control group. Absence of these risks factors might be an outcome of the short duration of our study.

In their study of 377 obstructive jaundice cases associated with malignancy, Dixon et al revealed that parameters including Hct levels lower than $30 \%$ and serum bilirubin levels higher than $20 \mathrm{mg} / \mathrm{dL}$ increased the risks of mortality and morbidity in such cases, and they also claimed that reducing serum bilirubin levels in the preoperative period could have been favorable for surgical outcomes [13]. Adverse effects of mechanical jaundice on renal, cardiovascular and immunologic functions have also been proven by other experimental studies [14]. Reduction of serum bilirubin levels results with decreased mortality and morbidity rates, and this concept attracted researchers' attention towards the hemodynamic and immunological affects that are caused by mechanical jaundice [14,15]. In the last decade, preoperative interventions for mechanical jaundice have resulted with better surgical outcomes [11]. This might be a result of proper patient selection, better preoperative preparation and improvements in treament.

It's known that hepatocyte functions fail, insufficiency in reticuloendothelial cell activity develops, and adverse metabolic changes occur in both organ function and wound healing in cases of mechanical jaundice $[9,14,15]$. Failure in hepatic functions has been detected in the present study as well. In the subgroup of subjects having undergone bile duct ligation, the remarkable histopathological alternations of the liver sections included dilation of the bile ducts and vessels, endothelial proliferation in the vessels lumens, periductal mesenchymal cell proliferation, lymphocyte, plasma cell and leukocyte infiltration, endothelial proliferation, vacuolization of hepatocytes, degenerative changes, and dilated sinusoids with erythrocyte accumulation all of which reveal the adverse effects of extrahepatic cholestasis on the metabolism and histology of the liver.

On the other hand, the prolonged use of octreotide has been reported to decrease bile flow and cause extrahepatic cholestasis in $20 \%$ of cases $[14,16]$. 
Therefore, we kept the observation period of our study as short as seven days to avoid this complication.

It is a known fact that free oxygen radicals are responsible for many negative events in mechanical jaundice $[5,17]$. The removal or neutralization of free oxygen radicals may play a role in improved outcomes. Therefore, we have included allopurinol in our study which also poses neutralizing effects on free oxygen radicals. However, four subjects in Group IV (CBDL+A) were found to have increased serum amylase levels and the histopathologic evaluations of the pancreatic samples of these four subjects revealed findings of pancreatitis.

In a study in which the antioxidant defense system in the liver was observed in experimental animals having undergone bile duct ligation, decreased vitamin $\mathrm{E}$ and selenium levels were found along with significant decrease in glutathione peroxidase activities and increased lipid peroxidation emphasizing the importance of oxidant damage in jaundice [4]. In mechaninal jaundice, increased levels of bilirubin and bile salts were blamed to distrupt cellular metabolism and membrane transport systems also causing a decrease in the cellular respiratory rate $[6,18]$. Additionally, it was reported that bile salts inhibit ATPase activity, thereby impairing lysosomal acivities [6]. In our study, it has been considered that although octreotide has beneficial effects on this process, allopurinol may have increased the oxidative damage in mechanical jaundice.

In mechanical jaundice, in addition to the negative effects of intestinal bacterial overgrowth, because of the excess load of ammonia that the liver is obliged to deaminate, the development of liver failure and increased levels of bile salts leads to the translocation of bacteria as well as endotoxemia $[2,16]$. In our study, we found that octreotide yielded at least promising results on liver functions.

\section{Conclusions}

We found no significant difference in biochemical, hematological and histopathological examinations except for serum direct bilirubin, ALT and AST levels between Group II (CBDL) and Group III (CBDL+O), which revealed that the short term application of octretoide posed some beneficial, however, not complete corrective effects on the hepatic changes caused by mechanical jaundice. On the other hand, in the treatment of fistula as a complication of biliary tract surgery (hepaticojejunostomy, choledochoduodenostomy, choledochojejunostomy, etc.) it can be safely used without additional hepatic side effects.

Although the general use of allopurinol for prophylactic or therapeutic purposes is likely to neutralize free oxygen radicals, it has been observed that 
allopurinol does not have a curative effect on the systemic consequences of mechanical jaundice. Additionally, since allopurinol increases the risk of pancreatitis, it has been considered that allopurinol should not be used for the treatment of surgical jaundice and that it should be used carefully for the aim of reducing the side effects of chemotherapy administered for biliary tract malignancies.

In conclusion, significantly decreased serum direct bilirubin, ALT and AST levels were detected with the application of octreotide in experimentally created mechanical jaundice. We believe that further well designed experimental and clinical studies are necessary to support our findings.

\section{Acknowledgements}

The authors would like to thank Orhan ALIMOGLU, MD, Prof.1 for his valuable contributions to this study.

1Department of General Surgery, Istanbul Medeniyet University, Faculty of Medicine, Goztepe Training \& Research Hospital, Istanbul, Turkey.

\section{References}

1. Ratych ER, SmithWG. Anatomy and physiology of the liver. In: George D, Zuidema GE (editors). Surgery of the alimentary tract. 4th ed, Philedelphia: W.B. Saunders Company; 1996. p. 357-74.

2. Ogata $\mathrm{Y}$, Nishi M, Nakayama H, Kuwahara T, Ohnishi Y, Tashiro S. Role of bile in intestinal barrier function and its inhibitory effect on bacterial translocation in obstructive jaundice in rats. J Surg Res 2003;115(1):18-23.

3. Abdeldayem H, Ghoneim E, Refaei AA, Abou-Gabal A. Obstructive jaundice promotes intestinal-barrier dysfunction and bacterial translocation: experimental study. Hepatol Int 2007;1(4):444-448.

4. Lamberts SW, Krenning EP, Klijn JG, Reubi JC. Clinical applications of somatostatin analogs. Trends Endocrinol Metab 1990;1(3):139-144.

5. Hirano T, Manabe T, Steer M, Printz $\mathrm{H}$, Calne R, Tobe T. Protective effects of therapy with a protease and xanthine oxidase inhibitor in short form pancreatic biliary obstruction and ischemia in rats. Surg Gynecol Obstet 1993;176(4):371-381.

6. Bailey ME. Endotoxin, bile salts and renal function in obstructive jaundice. $\mathrm{Br} \mathbf{J}$ Surg 1976;63(10):774-778.

7. Ohshio G, Saluja A, Steer ML. Effects of short-term pancreatic duct obstruction in rats. Gastroenterology 1991;100(1):196-202.

8. Liu XX, Sun SW, Yuan WJ, Gao H, Si YY, Liu K, Zhang S, Liu Y, Wang W. Isolation of Tricin as a Xanthine Oxidase Inhibitor from Sweet White Clover (Melilotus albus) and Its Distribution in Selected Gramineae Species. Molecules. 2018;23(10). pii: E2719.

9. Wait RB, Kahng KU. Renal failure complicating obstructive jaundice. Am J Surg 1989;157(2):256-263.

10. Togawa $\mathrm{O}$, Isayama $\mathrm{H}$, Tsujino $\mathrm{T}$, Nakai Y, Kogure H, Hamada T, Sasaki T, Yashima Y, Yagioka H, Arizumi T, Ito Y, 
Matsubara S, Yamamoto N, Sasahira N, Hirano K, Toda N, Tada M, Koike K. Management of dysfunctional covered selfexpandable metallic stents in patients with malignant distal biliary obstruction. J Gastroenterol 2013;48(11):1300-1307.

11. Pitt HA, Gomes AS, Lois JF, Mann LL, Deutsch LS, Longmire WP Jr. Does preoperative percutaneous biliary drainage reduce operative risk or increase hospital cost? Ann Surg 1985;201(5):545-553.

12. Blamey SL, Fearon KC, Gilmour WH, Osborne DH, Carter DC. Prediction of risk in biliary surgery. $\mathrm{Br} \mathrm{J}$ Surg 1983;70(9):535-538.

13. Dixon JM, Armstrong CP, Duffy SW, Davies GC. Factors affecting morbidity and mortality after surgery for obstructive jaundice: a review of 373 patients. Gut 1983;24(9):845-852.
14. Ding JW, Andersson R, Norgren L, Stenram U, Bengmark S. The influence of biliary obstruction and sepsis on reticuloendothelial function in rats. Eur $\mathbf{J}$ Surg 1992;158(3):157-164.

15. Pain JA. Reticulo-endothelial function in obstructive jaundice. Br J Surg 1987;74(12):1091-1094.

16. Cahill CJ, Pain JA, Bailey ME. Bile salts, endotoxin and renal function in obstructive jaundice. Surg Gynecol Obstet 1987;165(6):519-522.

17. Savdan M, Çakır M, Vatansev H, Küçükkartallar T, Tekin A, Tavlı Ş. Preventing oxygen free radical damage by proanthocyanidin in obstructive jaundice. Turk J Surg 2017;33(2):62-68.

18. Celik VK, Eken IE, Yildiz G, Yilmaz MB, Gurlek A, Aydin H. Vitamin E and antioxidant activity; its role in slow coronary flow. Cardiovasc J Afr 2013;24:360-363. 